\title{
Philosophiques
}

\section{Principes normatifs ou pragmatiques, et pondération des arguments}

\section{Pierre Livet}

Volume 31, numéro 2, automne 2004

URI : https://id.erudit.org/iderudit/009815ar

DOI : https://doi.org/10.7202/009815ar

Aller au sommaire du numéro

Éditeur(s)

Société de philosophie du Québec

ISSN

0316-2923 (imprimé)

1492-1391 (numérique)

Découvrir la revue

Citer ce document

Livet, P. (2004). Principes normatifs ou pragmatiques, et pondération des arguments. Philosophiques, 31(2), 402-405. https://doi.org/10.7202/009815ar d'utilisation que vous pouvez consulter en ligne.

https://apropos.erudit.org/fr/usagers/politique-dutilisation/ 
actions intentionnelles, nous ne réussissons à faire quelque chose - à traverser la rue, à lever la main - que si les circonstances sont favorables, si nous avons par exemple l'usage de nos membres. Quant à la croyance, il ne suffit pas de vouloir croire pour croire effectivement, cela est sûr. Mais il est dans notre pouvoir de croire quelque chose si nous prêtons attention aux raisons qui le justifient, et c'est dans ce sens qu'on dit couramment et à juste titre qu'un individu devrait croire ceci ou cela. Ici comme ailleurs, la vérité se trouve du côté d'un normativisme global.

\section{Principes normatifs ou pragmatiques, et pondération des arguments PIERRE LIVET \\ Université d'Aix-en-Provence}

Ruwen Ogien adopte généralement la stratégie suivante (dans ce livre comme dans les précédents) : il prend à chaque fois une thèse forte (internalisme des raisons morales, modularité massive, évolutionnisme, plaidoyer pour ne pas éliminer l'acrasie comme simple absence de rationalité, etc.); il montre que les arguments avancés en faveur de cette thèse forte sont attaquables. Il sousentend qu'une thèse forte devrait avoir des arguments forts, capables non seulement de résister à ces attaques, mais de les réduire à néant. Il suggère alors que la thèse forte n'a pas les moyens de ses prétentions, et que l'on pourrait donc envisager comme plus plausible une version (qui devra être faible) de la thèse qui inspire les contre-arguments.

Je me trouve en parfait accord avec la plupart des thèses qui ont la faveur de Ruwen Ogien (réalisme moral modéré, différence entre les valeurs et les normes tenant au rapport des normes à l'action, etc.). Mes critiques ne pourront donc porter que sur deux points : la thèse forte soutenue dans ce livre par Ruwen Ogien, selon laquelle nous avons besoin en éthique de métanormes comme «devoir implique pouvoir», comme un principe de parcimonie ( «inutile d'obliger les gens à faire ce qu'ils désirent déjà faire, inévitablement»); les insuffisances de la méthode que je viens de rappeler.

I. Le principe de «devoir implique pouvoir» ne me parait pas permettre un bon traitement de ce qu'on appelle les cas d'obligation contraire au devoir. Ainsi, quand j'ai heurté quelqu'un, je dois m'excuser. Or ma première obligation est de ne pas heurter mes voisins. Une fois que j'ai agi contrairement à cette obligation, je dois m'excuser. Évidemment je ne peux plus satisfaire l'obligation intiale. Celle-ci est-elle pour autant absente? Il ne le semble pas. Ruwen Ogien utilise des situations de ce genre pour conclure que «devoir implique pouvoir» n'est pas une implication conceptuelle, mais bien un principe normatif. Mais ce principe pris à la lettre n'est pas correct. Dans la situation évoquée, mon obligation subsiste, pour la pratique passée et pour la pratique future, et surtout, elle a même encore 
un rôle quant au présent. Elle guide ma révision de l'ordre des obligations. Si je fais passer l'obligation de m'excuser (qui n'était même pas présente avant que j'aie heurté cette personne) avant l'obligation de ne pas heurter, je ne mets cette obligation qu'à un second rang provisoire. Je contracte ainsi une obligation dérivée, qui consiste à remettre les choses en ordre une fois que je me suis excusé. Mais au moment où je heurte mon voisin, je suis non seulement dans l'obligation de m'excuser, ce qui implique de faire passer temporairement au premier rang cette obligation de m'excuser qui était très marginale, voire absente jusque là, mais aussi dans l'obligation de restaurer dans le futur immédiat (y compris au moment où je fais mes excuses) à son premier rang l'obligation de ne pas heurter mon voisin. Ainsi, au seul moment où l'obligation initiale est suspendue par le fait (j'ai heurté mon voisin), donc au moment où je ne peux pas la satisfaire, elle a encore cependant un rôle normatif, et elle reste donc un devoir, qui, bloqué dans son application pratique immédiate, a cependant toujours un rôle dans la révision de mon agenda d'obligations.

«Devoir implique pouvoir» ne peut donc pas être une méta-norme, c'est-à-dire une norme morale qui sélectionne toutes les normes candidates à être des normes morales. C'est simplement une norme pragmatique, qui donne à des normes morales temporairement bloquées dans leur application un autre rôle, celui de guider la révision de l'agenda de nos obligations.

Le principe de parcimonie n'a aussi que ce même statut de norme pragmatique. Ruwen Ogien le reconnaît d'ailleurs. Ce que nous désirons déjà faire, il est inutile de nous l'imposer comme obligation. Certes, c'est inutile, mais ce n'est pas immoral. Ruwen Ogien admet que «obliger quelqu'un à faire ce qu'il veut ne serait pas moralement injuste (p. 91)». Mais alors ce principe de parcimonie n'est pas une méta-norme morale. Sinon, dès que je désirerais effectivement et inévitablement quelque chose, sa qualification morale d'obligation disparaîtrait. Or la solution de ce problème est la même que précédemment : parfois l'obligation n'a pas à avoir d'effet pratique en tant qu'obligation, puisqu'il est impossible de discerner cet effet pratique de celui du commandement de l'obligation. Ici, au lieu de bloquer l'application de l'obligation, on la rend indiscernable de l'effet de nos désirs. Mais cela ne doit nullement éliminer son rôle de guide dans l'agenda de nos devoirs.

Nous n'avons donc pas affaire à deux principes, mais au même, et qui est en un sens opposé aux deux formulations "devoir implique pouvoir ", et "il est inutile d'ordonner à quelqu'un ce qu'il veut». Le mieux serait de le formuler comme un principe de priorité du moral sur le pragmatique, même en cas d'impossibilité pragmatique : les conditions pragmatiques ne sont pas de nature à éliminer des obligations morales, mais au plus à détourner temporairement les modalités de leur influence.

Il n'est même pas possible d'éliminer le rôle purement épistémique de ce principe, qui consiste à mentionner l'existence, dans la liste de 
nos obligations, de ce devoir, sous prétexte qu'il faudrait appliquer un rasoir d'Occam dans le domaine kantien. Ne plus mentionner ce devoir parce qu'il est appliqué en toutes circonstances serait en fait changer notre accès épistémique à la hiérarchie des valeurs, et cela pourrait avoir des conséquences désastreuses sur le soutien à d'autres devoirs, qui, eux, ne sont pas inévitablement satisfaits.

Le principe de parcimonie se justifie comme principe moral seulement si on le comprend dans le cadre du problème du mode de publicité des obligations morales. Devant des acteurs qui satisfont les obligations morales (peut-être en suivant leurs désirs, peut-être en suivant des obligations, mais toujours librement), nous n'avons pas à leur rappeler prétentieusement et sans cesse qu'ils doivent remplir ces devoirs. Mais c'est alors simplement un principe d'une société «décente», qui évite autant que possible les humiliations injustifiées.

II. Comme exemple des incertitudes auxquelles peut mener la méthode utilisée généralement par Ruwen Ogien, je citerai ses arguments sceptiques vis-à-vis des tentatives d'explication évolutionniste des sentiments moraux (même si je partage ce scepticisme). Contre Gibbard, Ogien rappelle que nous sommes incapables de faire un bilan complet de ce que pourraient être les avantages pour la survie et le développement d'une société de son utilisation des rapports complémentaires entre la honte et la culpabilité d'un côté, le ressentiment de l'autre. En revanche, contre l'idée d'un "module de détection des tricheurs" de Tooby et Cosmides, Ogien rappelle qu'il y a tout de même $25 \%$ de personnes qui se trompent dans le test proposé, et que la présence d'une capacité à détecter les tricheurs dans $75 \%$ de la population est insuffisante pour supposer un mécanisme de sélection évolutive. Ce mécanisme devrait être efficace à $100 \%$ (p. 173). C'est une erreur. Des combinaisons de diverses fréquences de sous-populations (du type $75 \%-25 \%$ ) sont souvent des stratégies évolutionnairement stables.

Les explications évolutionnaires peuvent avoir deux rôles opposés, qui n'impliquent pas les mêmes exigences. Le premier, très exigeant et très rarement rempli, est de fournir un mécanisme évolutionnaire dont on puisse apporter des preuves qu'il a réellement fonctionné. Le second, à l'autre extrême, est simplement de construire un modèle stylisé qui montre la possibilité d'une explication évolutionnaire pour rendre compte par des concepts moins exigeants de situations décrites dans les termes de concepts plus exigeants. Gibbard propose un dosage très subtil d'interactions, d'émotions, et de publicité collective de la reconnaissance de normes (toutes choses descriptives, même si cette confession de reconnaissance des normes fait référence à du normatif), et ce dosage a cette fonction conceptuelle. Il est curieux que Ruwen Ogien lui oppose l'impossibilité d'un calcul effectif (dans le monde réel), alors que par ailleurs 
il accepte ce qu'il nomme «l'argument sophistique " : s'il est raisonnable de parfois agir selon son meilleur jugement, c'est parce que parfois cette action se trouve en fait - aux yeux de tiers - rationnelle. Mais la probabilité que nous nous trouvions dans de telles situations est très faible.

À tout le moins, on ne peut l'encourager que dans beaucoup moins de $25 \%$ des cas, et elle ne pourrait donc être rationalisée que par une stratégie évolutionnairement stable qui soit faite d'une mixture de probabilités. Je ne suis pas un adepte de l'utilisation du formalisme des probabilités dans ces domaines, mais, à tout le moins, il serait raisonnable de ne pas ranger toutes les normes morales auxquelles on peut penser au même rang de normalité. Ce sont des réflexions sur ces pondérations - pondérations des arguments et pondération des normes - qui me semblent faire défaut dans la méthode de Ruwen Ogien, alors même que ses conclusions montrent que la plupart du temps il pratique sans la thématiser une telle pondération.

\section{Rationalité, humanité, normativité}

\section{SARAH STROUD McGill}

Ce livre plein de sagesse, de modération, de bon sens donne au lecteur le sentiment agréable de participer à un dialogue philosophique avec l'auteur. Dans l'esprit d'une telle conversation, j'aimerais poser ici quelques questions suscitées par certains des essais colligés dans Le rasoir de Kant. Elles ont pour but de poursuivre le dialogue avec Ruwen Ogien que ses réflexions dans le livre semblent solliciter.

\section{Le rationnel et le raisonnable; la partialité et l'impartialité}

Ogien lie le rationnel à la partialité et le raisonnable à l'impartialité. Ce premier article du recueil soulève donc la question de la relation entre la partialité et l'impartialité. J'aimerais souligner une remarque importante de l'auteur à ce sujet. Bon nombre de critiques de l'impartialité en éthique sont en fait dirigées contre l'idée d'un "principe substantiel» (32) d'impartialité qui nous ordonnerait de traiter chaque personne de la même façon ${ }^{1}$. Mais, comme le mentionne Ogien, ce n'est pas le seul rôle que peut jouer l'impartialité dans un système éthique; et il est frappant de constater que peu de théories morales courantes contiennent un tel principe de premier ordre. Passons donc à l'idée que l'impartialité est plutôt un point de vue à partir duquel il est possible de choisir, d'adopter, d'endosser ou de justifier des principes de premier ordre. Ogien remarque que le fait d'occuper une telle perspective n'implique pas

1. Voir par exemple John Cottingham, «Ethics and Impartiality ", Philosophical Studies 43 (1983), 83-99; John Kekes, "Morality and Impartiality ", American Philosophical Quarterly 18 (1981), 295-303; Lawrence Blum, Friendship, Altruism, and Morality (Boston : Routledge \& Kegan Paul, 1980), ch. III. 